\title{
Erratum to: The crystal structure of Sporosarcina pasteurii urease in a complex with citrate provides new hints for inhibitor design
}

\author{
Stefano Benini • Paulina Kosikowska • \\ Michele Cianci - Luca Mazzei - Antonio Gonzalez Vara • \\ Lukasz Berlicki $\cdot$ Stefano Ciurli
}

Published online: 14 March 2013

(C) SBIC 2013

Erratum to: J Biol Inorg Chem (2013) 18:391-399

DOI 10.1007/s00775-013-0983-7

The original publication of the article includes an error in the last sentence of Acknowledgement section.

The correct sentence should read as "The structure was deposited in the RCSB Protein Data Bank with accession code 4AC7."

The online version of the original article can be found under doi:10.1007/s00775-013-0983-7.

\section{S. Benini}

Faculty of Science and Technology,

Free University of Bolzano, Bolzano, Italy

P. Kosikowska $\cdot$ Ł. Berlicki

Department of Bioorganic Chemistry,

Wrocław University of Technology,

Wrocław, Poland

M. Cianci

European Molecular Biology Laboratory c/o DESY,

22603 Hamburg, Germany

L. Mazzei · S. Ciurli $(\bowtie)$

Laboratory of Bioinorganic Chemistry,

Department of Pharmacy and Biotechnology,

University of Bologna, Via Fanin 40,

40127 Bologna, Italy

e-mail: stefano.ciurli@unibo.it

A. G. Vara

Department of Industrial Chemistry,

University of Bologna, Bologna, Italy 